\title{
Socio-cultural determinants of anticipated acceptance of an oral cholera vaccine in Western Kenya
}

\author{
N. SUNDARAM ${ }^{1,2 *}$, C. SCHAETTI ${ }^{1,2}$, C.-L. CHAIGNAT ${ }^{3}$, R. HUTUBESSY $^{4}$, \\ E. O. NYAMBEDHA ${ }^{5}$, L. A. MBONGA ${ }^{5}$ AND M. G. WEISS ${ }^{1,2}$ \\ ${ }^{1}$ Department of Epidemiology and Public Health, Swiss Tropical and Public Health Institute, Basel, Switzerland \\ ${ }^{2}$ University of Basel, Basel, Switzerland \\ ${ }^{3}$ Global Task Force on Cholera Control, World Health Organization, Geneva, Switzerland \\ ${ }^{4}$ Initiative for Vaccine Research, World Health Organization, Geneva, Switzerland \\ ${ }^{5}$ Department of Sociology and Anthropology, Maseno University, Kisumu, Kenya
}

Received 16 November 2011; Final revision 17 March 2012; Accepted 10 April 2012; first published online 8 May 2012

\section{SUMMARY}

Determinants of anticipated acceptance of an oral cholera vaccine (OCV) were studied in urban and rural communities of Western Kenya. An explanatory model interview administered to 379 community residents assessed anticipated vaccine acceptance at various prices from no cost to full-cost recovery, socio-cultural features of cholera and social characteristics. Nearly all (99\%) residents indicated willingness to accept a no-cost OCV, $95 \%$ at a price of US $\$ 0 \cdot 8,73 \%$ at US\$ $4 \cdot 2$ and $59 \%$ at US\$ 8.4. Logistic regression models analysed socio-cultural determinants of anticipated OCV acceptance. Prominence of non-specific symptoms for cholera was negatively associated with acceptance. A cholera-specific symptom (thirst), self-help referring to prayer, income and education were positively associated. In the high-cost model, education was no longer significant and reliance on herbal treatment was a significant determinant of vaccine nonacceptance. Findings suggest high motivation for OCVs, if affordable. Socio-cultural determinants are better predictors of anticipated acceptance than socio-demographic factors alone.

Key words: Cholera, Kenya, oral cholera vaccine, social and cultural determinants, vaccine acceptance.

\section{INTRODUCTION}

Among infectious diseases, diarrhoeal diseases rank as the third leading cause of mortality and morbidity in low- and middle-income countries [1]. It is estimated that diarrhoeal diseases account for 1.78 million

\footnotetext{
* Author for correspondence: Ms. N. Sundaram, Department of Epidemiology and Public Health, Swiss Tropical and Public Health Institute, Socinstrasse 57, CH-4002 Basel, Switzerland. (Email: neisha.sundaram@stud.unibas.ch)
}

deaths per year and 58.7 million disability-adjusted life years. Cholera, a rapidly dehydrating diarrhoeal disease, is estimated to cause the death of 100000 130000 persons and account for 3-5 million cases per year [2]. Kenya suffers from a high burden of cholera, having reported 11425 cases and 264 deaths in 2009 [3].

Cholera transmission is closely associated with environmental conditions, spread by faecal contamination of water and food [4]. Access to safe water and 
adequate sanitation are fundamentals of cholera prevention. In many countries, however, implementing relevant measures has proved difficult and slow [2,5]. Furthermore, improvements may not reach the most vulnerable populations, such as those living in slums and remote rural areas, in the near future [5]. Vaccines may therefore have a critical role as a provisional public health tool in cholera control in these communities. In 2005, the World Health Organization (WHO) first suggested oral cholera vaccines (OCVs) be used in cholera-endemic areas as a supplementary control strategy [6] and they strengthened that recommendation in 2010 [4].

Two OCVs are currently pre-qualified by the WHO for international use. Both Dukoral ${ }^{\circledR}$ (Crucell, The Netherlands), containing recombinant cholera toxin B subunit and killed whole-cell $V$. cholerae $\mathrm{O} 1$, and Shanchol $^{\mathrm{TM}}$ (Shantha Biotechnics Ltd, India), containing killed $V$. cholerae $\mathrm{O} 1$ and $\mathrm{O} 139$, have been shown to be efficacious in endemic settings [7, 8]. Although safety, efficacy and an efficient health system to distribute the vaccine are critical, understanding cultural preferences and the willingness of communities to accept the vaccine are also essential. Assessing socio-cultural features of the illness and willingness to accept a vaccine indicate perceived need, demand and cultural barriers that may reduce coverage in a vaccine campaign. Notwithstanding recognized value of such research [9, 10], studies have been largely confined to high-income countries [11, 12]. Cholera vaccine acceptance studies focus mainly on sociodemographics and willingness to pay [13-15], while studies that have considered socio-cultural aspects of cholera have concentrated on Asia [16, 17]. Research is lacking on cultural dimensions and social determinants of cholera vaccine acceptance in Kenya.

This study was conducted in Nyanza province of Western Kenya due to the disproportionately high number of cholera cases reported there compared to the rest of Kenya [18]. Two large cholera outbreaks occurred there in 1997-1998 and 2008 that accounted for $43-47 \%$ and $72 \%$, respectively, of all cholera cases in Kenya [19, 20]. Urban and rural sites were chosen because they differ significantly in terms of environmental conditions, population density, residents' income and occupation; the implication being that the findings from one setting may not be attributable to the other. Cultural epidemiological methods [21] were employed to understand community experience, meaning and behaviour with a cholera-like illness. The objectives of this paper are to (a) assess community willingness to accept an OCV in urban and rural populations in Western Kenya, (b) analyse socio-cultural determinants of anticipated OCV acceptance and $(c)$ clarify the role of sociocultural features of illness in explaining anticipated OCV acceptance by comparing models that consider socio-cultural determinants with exclusively sociodemographic models.

\section{METHODS}

\section{Setting}

This study was conducted at both urban and rural sites in Nyanza province, Western Kenya, where cholera is considered endemic. The urban site at Nyalenda A, Winam division, Kisumu district is a heavily populated informal settlement and the rural site is comprised of villages at Kakum Kombewa sublocation, Boro division, Siaya district.

The urban site covers an area of $2.8 \mathrm{~km}^{2}$, has 23731 residents and a population density of 8475 persons/ $\mathrm{km}^{2}$ [22]. The majority of residents do not have access to piped water and largely rely on shallow wells that are subject to a high level of contamination due to the predominance of pit latrines [22,23]. There are no government health facilities in Nyalenda $\mathrm{A}$ and private health services involve higher costs to be borne.

The rural site is comprised of nine villages at Kakum Kombewa with a population density of around 270 individuals $/ \mathrm{km}^{2}$ [24]. Main sources of water in this region are untreated streams and boreholes. The majority $(73 \%)$ of the population have access to latrines; however, over $24 \%$ of these are in a poor state and hence not used [24]. Lack of public transport makes access to Siaya district hospital, which is located about $15 \mathrm{~km}$ away, difficult.

\section{Study design and sampling}

This cross-sectional study required a minimum sample size of 328 to allow for cross-site comparisons with $95 \%$ significance and $80 \%$ power [25]. Men and women from the general population between the ages of 18 and mid-60s were included.

At the urban site, only an estimate of the population size was obtainable, hence, systematic probability sampling was done. The area was divided into seven roughly equal segments and every fifth household was approached to get a total of 28 households 
per segment. At the rural site, detailed household lists were accessible through community health workers. A specific number of households per village, proportional to the total number of households in that village, which had been identified in advance through random selection, were approached. At both sites, one willing adult of the household was interviewed; selection was made to maintain a roughly equal balance between men and women. When more than one eligible adult was available, we asked them to decide whom we should interview. If a household had no suitable, willing candidate, the neighbouring household was approached.

\section{Instrument and data collection}

This study used a semi-structured explanatory model interview based on the framework of the Explanatory Model Interview Catalogue (EMIC) for cultural epidemiology [26]. It was developed for the study of cholera to assess locally valid features of illnessrelated experience, meaning and behaviour from the perspective of community residents [27]. The illness was introduced to participants using a clinical vignette that described a person with physical symptoms of cholera. Respondents were asked what they would call such an illness and the term for the illness provided by the respondent was used when asking further questions. In addition to questions on socio-cultural features of illness (i.e. physical symptoms, social impact, perceived causes, help-seeking behaviour), the interview also included questions on respondents' socio-demographic characteristics and their ideas on general vaccination. Quantitative and qualitative data were both collected.

Respondents were also asked if they would be willing to take a vaccine that is swallowed to prevent cholera. Details of efficacy and duration of protection were not discussed. OCV acceptance questions were posed at four different prices: 'high', based on estimated full production cost recovery for manufacture of two doses of Dukoral (KES 650/US\$ 8.4) †; 'medium', which is half the high price (KES 325/US\$ $4 \cdot 2)$; 'low', close to the US\$ 1 price that is considered a realistic vaccine price for low- and middle-income countries (KES 65/US\$ 0.8) [16] and 'free', fully subsidized as in the case of many immunization campaigns.

$\dagger$ Exchange rate: Kenya shilling (KES) $1=$ US\$ 0.01287 as of 1 March 2010 (www.oanda.com).
Interviews were conducted between March and May 2010, in Kiswahili, Dholuo and English. Interviewers received extensive training in sampling procedures, interviewing and obtaining informed consent. The interviewers were science or social science graduates from Maseno University and introduced themselves accordingly. Interviews were voice-recorded with permission.

\section{Data management and analysis}

EMIC interview data were double-entered using Epi Info software version 3.5.1 (Centers for Disease Control and Prevention, USA), programmed with logic and range checks. For analysis of socio-cultural features of illness, prominence of categories was calculated based on whether a response was reported spontaneously (assigned value of 2) or after probing (assigned value of 1). When a category was identified as most important among all others, it was assigned an additional value of 3 . A mean prominence was then calculated for each category. Through this method of prominence calculation, categories were evaluated based on relative importance ascribed to them by local cultural ideas.

Logistic regression analyses were done to empirically identify socio-cultural determinants (i.e. sociocultural features of illness and socio-demographic characteristics) associated with anticipated $\mathrm{OCV}$ acceptance at various prices. Dichotomized anticipated OCV acceptance variables, reflecting vaccine acceptance or non-acceptance, were used as outcome variables. Separate regression analyses were performed for anticipated OCV acceptance at the medium price and at the high price, but not for the low price or no-cost models as acceptance rates over $95 \%$ did not allow for it.

In crude analysis, associations between OCV acceptance and explanatory variables that were reported by $5-95 \%$ of respondents were analysed. Variables with $P<0.2$ were considered for multivariate analysis. 'Focal' models of socio-cultural features of illness for specific groups of variables (i.e. related to physical symptoms, social impact, perceived causes, help-seeking), adjusted for sociodemographic variables, were run. Focal models for socio-demographic factors alone were also considered. Interaction of site with each of the variables was tested and site-interaction terms with $P<0 \cdot 1$ were included. To estimate the combined influence of all categories identified in the focal models on 
anticipated OCV acceptance, a 'comprehensive' model was calculated using variables with $P<0 \cdot 2$ and site-interaction terms with $P<0 \cdot 1$ from focal models. Corrected Akaike's Information Criterion (AICc) values were computed to compare relative goodness of fit in various focal and comprehensive regression models. $\triangle(\mathrm{AICc})$ which represents the difference in AICc between each model and the model with the lowest AICc, was used to make this comparison. Models with lower $\triangle$ (AICc) values are considered better in explaining OCV acceptance than those with higher values. Quantitative analysis was done with SAS version 9.2 (SAS Institute Inc., USA).

Narrative accounts were translated into English and entered in word processor software. Additional detail from key questions of the interview was added by transcribing relevant voice records. Typed data were then imported into MAXQDA version 10 (VERBI Software, Germany) for qualitative data management and analysis. Text segments were thematically coded based on the interview structure. Variables were imported into MAXQDA to enable the selection of narrative records of interest based on results from the quantitative analysis. This approach enabled integrated analysis of quantitative and qualitative data.

\section{Ethical considerations}

The study protocol received ethical approval from the Kenya Medical Research Institute and the WHO Research Ethics Review Committee. Interviews were conducted after obtaining written informed consent. No financial or other incentives were provided to respondents. Data collected in this study was maintained with utmost confidentiality and anonymized for reporting.

\section{RESULTS}

\section{Sample characteristics}

Of 379 respondents interviewed, $50 \%$ were from the urban site and $51 \%$ were female (Table 1). All respondents at the rural site and $96.8 \%$ at the urban site identified Christianity as their religion. The median personal monthly income was KES 2500 (US\$ 32) at the urban site and KES 1000 (US\$ 13) at the rural site $(P=0 \cdot 01)$. Significantly more respondents at the urban site reported a dependable source of income.
Self-employment (e.g. petty trading and skilled labour) was the most frequently mentioned primary occupation at the urban site; and agriculture at the rural site. Urban respondents were better educated: more in the urban sample had a secondary education or higher; more in the rural sample had no education. The rural site had significantly more individuals living within a household than at the urban site.

\section{Past experience with vaccination and general ideas on vaccines}

Two-thirds $(66 \cdot 8 \%)$ of all respondents reported having personally received a vaccination in the past. Fewer respondents reported prior vaccination experience at the rural $(51.3 \%)$ than at the urban $(82 \cdot 1 \%, P<0 \cdot 001)$ site.

All but four respondents $(98.9 \%)$ stated that in their experience, vaccines were helpful. The idea that vaccines were beneficial in preventing disease was reported pervasively. More knowledgeable respondents provided accounts with a scientific basis, such as, 'Vaccines are helpful; they boost the immune system and prevent future infections' (urban woman, 22 years). There also seemed to be a high level of confidence in the protective effect of vaccines, as seen in this narrative, 'I rarely get sick because I was vaccinated' (urban man, 30 years).

When asked whether some vaccines were also likely to cause problems, $27 \cdot 7 \%$ of the respondents agreed; pain at the injection site, infection/abscess, fever and disability were frequently cited problems. Of respondents who believed that vaccines were not likely to cause problems, there was a significant difference in terms of site $(P=0.036)$ and gender $(P=0.007)$, with more women and more urban respondents espousing this view.

\section{Anticipated OCV acceptance}

Almost all respondents $(98.7 \%)$ reported an interest in accepting an OCV if it were to be made available free of charge (Fig. 1). At the low price, $95 \cdot 3 \%$ respondents were willing to accept the vaccine. At the medium and high prices, $72 \cdot 8 \%$ and $58 \cdot 8 \%$ respondents, respectively, were interested in the vaccine. More urban than rural respondents were willing to accept an OCV at the medium $(P=0.008)$ and high $(P=0.002)$ prices. Anticipated OCV acceptance rates between men and women were similar. 
Table 1. Socio-demographic characteristics of study respondents

\begin{tabular}{|c|c|c|c|c|}
\hline & Overall $(n=379)$ & Urban $(n=190)$ & Rural $(n=189)$ & $P$ value $^{\mathrm{d}}$ \\
\hline \multicolumn{5}{|l|}{ Gender (\%) } \\
\hline Female & $51 \cdot 2$ & $52 \cdot 1$ & $50 \cdot 3$ & \\
\hline \multicolumn{5}{|l|}{ Age (years) } \\
\hline Mean (s.D.) $)^{\mathrm{a}}$ & $32 \cdot 8(13 \cdot 1)$ & $28 \cdot 9(10 \cdot 1)$ & $36 \cdot 8(14 \cdot 5)$ & $* * *$ \\
\hline Median (range) ${ }^{\mathrm{b}}$ & $29(18-69)$ & $25(18-63)$ & $33(18-69)$ & $* * *$ \\
\hline \multicolumn{5}{|l|}{ Household size (persons) } \\
\hline Mean (S.D.) $)^{\mathrm{a}}$ & $4 \cdot 5(2 \cdot 3)$ & $4 \cdot 3(2 \cdot 1)$ & $4 \cdot 7(2 \cdot 6)$ & $*$ \\
\hline \multicolumn{5}{|l|}{ Main occupation $(\%)^{\mathrm{c}}$} \\
\hline Agriculture & $25 \cdot 3$ & $1 \cdot 6$ & $49 \cdot 2$ & $* * *$ \\
\hline Self-employed & $26 \cdot 6$ & $36 \cdot 8$ & $16 \cdot 4$ & $* * *$ \\
\hline Formal employment & $12 \cdot 4$ & $16 \cdot 8$ & $7 \cdot 9$ & $*$ \\
\hline Housewife & $9 \cdot 0$ & $14 \cdot 7$ & $3 \cdot 2$ & $* * *$ \\
\hline Casual labourer & $9 \cdot 2$ & $12 \cdot 1$ & $6 \cdot 3$ & \\
\hline Student & $5 \cdot 5$ & $6 \cdot 3$ & $4 \cdot 8$ & \\
\hline Not active/retired & $9 \cdot 5$ & $10 \cdot 0$ & $9 \cdot 0$ & \\
\hline \multicolumn{5}{|c|}{ Highest education level attended $(\%)^{\mathrm{c}}$} \\
\hline No education & $3 \cdot 7$ & $0 \cdot 5$ & $6 \cdot 9$ & $* * *$ \\
\hline Primary school & $50 \cdot 1$ & $44 \cdot 7$ & $55 \cdot 6$ & $*$ \\
\hline Secondary school & $37 \cdot 7$ & $46 \cdot 8$ & $28 \cdot 6$ & $* * *$ \\
\hline Vocational school & $1 \cdot 8$ & $0 \cdot 0$ & $3 \cdot 7$ & $* *$ \\
\hline College and above & $6 \cdot 6$ & $7 \cdot 9$ & $5 \cdot 3$ & \\
\hline \multicolumn{5}{|l|}{ Household income $(\%)^{\mathrm{c}}$} \\
\hline Regular and dependable & $47 \cdot 8$ & $66 \cdot 8$ & $28 \cdot 6$ & $* * *$ \\
\hline
\end{tabular}

S.D., Standard deviation.

a $t$ test.

b Wilcoxon test.

${ }^{\mathrm{c}}$ Fisher's exact test. Only categories with overall reported percentages $>1.5 \%$ are displayed.

${ }^{d} P$ value obtained from a comparison between the urban and rural site; ${ }^{*} P \leqslant 0 \cdot 05 ; * * P \leqslant 0 \cdot 01 ; * * * P \leqslant 0 \cdot 001$.

Narrative accounts demonstrate an active demand for cholera vaccines. A sense of urgency in obtaining cholera vaccines was communicated as follows:

We have a water problem because there is a lot of pollution in the water and water points are scarce. We are also far from the hospital in Siaya. So I ask, when will this vaccine come? Or will you just disappear after the research? We really need the vaccine (rural man, 32 years).

A highly mentioned reason for willingness to purchase an OCV was that it would be more cost-effective than spending money on cholera treatment in the future.

Vaccine cost was a critical point of consideration for many respondents. While requesting a free vaccine, a 35-year-old rural woman explained:

If a vaccine is introduced, let it be free of charge so that it can help everyone. If it is brought with a price, others will die if they cannot afford it.

However, demand for a vaccine was high enough for respondents to offer suggestions that could enable vaccine purchase, even if it could not be availed for free.

If you bring the vaccine, tell us in advance so that we have enough time to collect money to pay for it. If you come without notice, we may not have the money ready (rural woman, 26 years).

The idea that health was more important than money was widespread and many stated: 'You cannot compare your life to money.'

\section{Determinants of anticipated $\mathrm{OCV}$ acceptance at the medium price and high price}

Focal regression models considered specific groups of explanatory variables in explaining anticipated OCV acceptance at the medium and high prices (Tables 2 and 3). As per their $\Delta$ (AICc) values, at the medium price, 'somatic symptoms' and 'selftreatment at home' models explained acceptance better than the focal model with only socio-demographic 


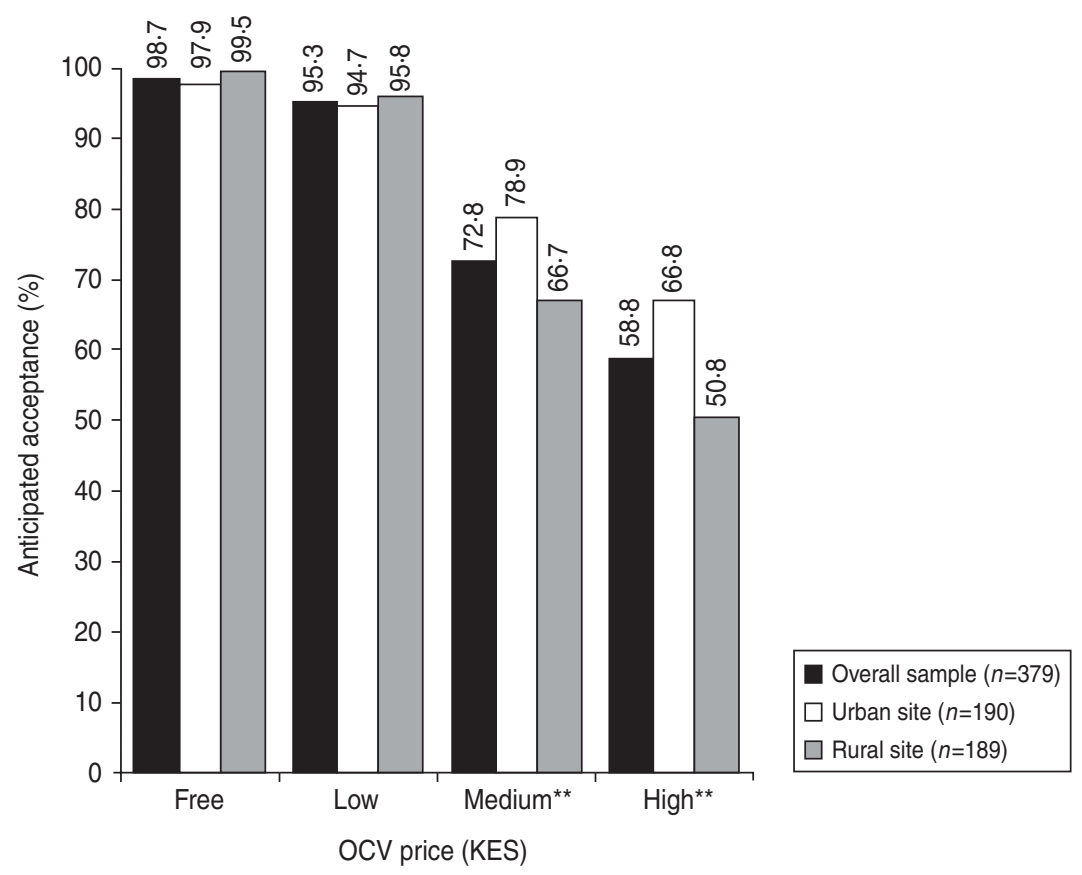

Fig. 1. Anticipated oral cholera vaccine (OCV) acceptance at different prices among urban and rural residents of Western Kenya. OCV price mentioned in Kenya shillings (KES) to respondents: low (KES 65/US\$ 0.8), medium (KES 325/US\$ 4.2), and high (KES 650/US\$ 8.4) (exchange rate: KES $1=$ US\$ 0.01287). Y axis denotes percentage of respondents who provided a favourable response when questioned on whether they were likely to buy the vaccine at the stated price. Fisher's exact test was used for comparison of percentages between the two sites. $* * P \leqslant 0 \cdot 01$.

characteristics. At the high price, all socio-cultural focal models were better than the exclusively sociodemographic model. Comprehensive models, which combined significant variables from all focal models, explained OCV acceptance best.

Most variables that were significant in the focal models remained so in the comprehensive models. Socio-cultural determinants identified in the comprehensive models that were associated with anticipated OCV acceptance at the medium price and the high price are shown in Tables 4 and 5, respectively. Although different explanatory variables were identified in the analyses at the two price levels, they refer to coherent themes explaining anticipated OCV acceptance: specificity of symptoms for cholera, level of education, restricted preference for treatment and financial viability.

Identification of physical symptoms that were unrelated to cholera such as bloody stool, and nonspecific for cholera such as loss of appetite and confusion, were negatively associated with OCV acceptance. A cholera-specific symptom of being 'very thirsty' was positively associated.

Having attended secondary school was positively associated with OCV acceptance at the medium price.
However, this did not remain significant at the high price.

With an increase in price of the vaccine from medium to high, the reporting of herbal treatment as a home remedy became significantly negatively associated with acceptance. In contrast, the reporting of prayer as a form of self-treatment at home was positively associated with OCV acceptance at both prices. An analysis of qualitative accounts revealed that prayer and medical interventions are considered complementary forms of treatment, carried out in parallel. Prayer and medicine are believed to have different, but non-conflicting roles, in treatment. 'Prayer must be conducted to have God's intervention while pharmacy drugs help in controlling the situation' (urban woman, 22 years). However, medical help was often implicitly assigned a greater priority while prayer was recommended in addition. Even a respondent who stated, 'Prayer helps because God is above everything, even above drugs', further mentioned that she would first give the patient water and drugs to combat diarrhoea, and thereafter pray.

Household income and household size significantly influenced OCV acceptance. The former was 
Table 2. Multivariate analysis (focal models) of socio-cultural determinants of anticipated oral cholera vaccine acceptance at the medium price (US\$ 4.2) and assessment of models

\begin{tabular}{|c|c|c|c|c|}
\hline Focal models ${ }^{\mathrm{a}}$ & Coefficient $(95 \% \mathrm{CI})^{\mathrm{b}}$ & $P$ value ${ }^{\mathrm{c}}$ & $\operatorname{Int}^{\mathrm{d}}$ & $\Delta(\mathrm{AICc})^{\mathrm{e}}$ \\
\hline Patterns of distress: somatic symptoms & & & & $3 \cdot 25$ \\
\hline Bloody stool (urban site) & $0 \cdot 35(-0 \cdot 30$ to $1 \cdot 00)$ & $0 \cdot 288$ & & \\
\hline Bloody stool (rural site) & $-0.43(-0.75$ to $-0 \cdot 11)$ & $0 \cdot 008$ & * & \\
\hline Very thirsty & $0.61(0.02$ to $1 \cdot 21)$ & $0 \cdot 044$ & & \\
\hline Loss of appetite & $-0.73(-1 \cdot 16$ to $-0 \cdot 31)$ & $0 \cdot 001$ & & \\
\hline Palpitations & $-0 \cdot 08(-0 \cdot 41$ to $0 \cdot 26)$ & $0 \cdot 652$ & & \\
\hline Confusion & $-0.52(-0.92$ to $-0 \cdot 12)$ & $0 \cdot 012$ & & \\
\hline Patterns of distress: social impact & & & & $22 \cdot 6$ \\
\hline Fear of infecting others & $0 \cdot 17(-0 \cdot 10$ to $0 \cdot 45)$ & $0 \cdot 217$ & & \\
\hline Perceived causes & & & & $24 \cdot 43$ \\
\hline Eating soil & $0.09(-0.41$ to 0.60$)$ & 0.723 & & \\
\hline Malaria & $-0.29(-0.87$ to 0.28$)$ & $0 \cdot 318$ & & \\
\hline Violation of taboo/tradition & $-0.17(-0.59$ to 0.26$)$ & $0 \cdot 437$ & & \\
\hline Other causes (urban site) ${ }^{\mathrm{f}}$ & $0.13(-0.28$ to 0.54$)$ & 0.527 & & \\
\hline Other causes (rural site) ${ }^{\mathrm{f}}$ & $-0 \cdot 37(-0.64$ to $-0 \cdot 09)$ & $0 \cdot 010$ & * & \\
\hline Cannot say & $-0 \cdot 15(-0 \cdot 34$ to $0 \cdot 05)$ & $0 \cdot 137$ & & \\
\hline Self-treatment at home & & & & $17 \cdot 27$ \\
\hline Drinking more water or liquids & $0 \cdot 11(-0 \cdot 06$ to $0 \cdot 28)$ & $0 \cdot 212$ & & \\
\hline Herbal treatment & $-0.23(-0.44$ to $-0 \cdot 01)$ & $0 \cdot 040$ & & \\
\hline Prayers & $0.43(0.07$ to 0.79$)$ & $0 \cdot 021$ & & \\
\hline Drink with alcohol & $0 \cdot 53(-0 \cdot 24$ to $1 \cdot 31)$ & $0 \cdot 178$ & & \\
\hline Socio-demographics ${ }^{\mathrm{g}}$ & & & & $22 \cdot 08$ \\
\hline Primary school vs. no education & $0.58(-0.57$ to 1.73$)$ & $0 \cdot 325$ & & \\
\hline Secondary school vs. no education & $1 \cdot 02(-0 \cdot 17$ to $2 \cdot 22)$ & $0 \cdot 093$ & & \\
\hline $\begin{array}{l}\text { Regular and dependable household income } \\
\text { (urban site) }\end{array}$ & $1.54(0.79$ to 2.30$)$ & $<0 \cdot 001$ & & \\
\hline $\begin{array}{l}\text { Regular and dependable household income } \\
\text { (rural site) }\end{array}$ & $0 \cdot 12(-0.58$ to $0 \cdot 82)$ & 0.739 & $* *$ & \\
\hline Household size & $-0.07(-0.17$ to 0.03$)$ & $0 \cdot 189$ & & \\
\hline Occupation: housewife, student, retired ${ }^{\mathrm{h}}$ & $-0.26(-0.98$ to 0.46$)$ & $0 \cdot 474$ & & \\
\hline $\begin{array}{l}\text { Occupation: self-employed, formally employed, } \\
\text { casual labour }^{\mathrm{h}}\end{array}$ & $0 \cdot 23(-0.42$ to 0.88$)$ & $0 \cdot 479$ & & \\
\hline Gender (male $v s$. female) & $0 \cdot 25(-0.26$ to $0 \cdot 76)$ & $0 \cdot 330$ & & \\
\hline Site (rural vs. urban) & $0 \cdot 36(-0 \cdot 35$ to $1 \cdot 07)$ & $0 \cdot 316$ & & \\
\hline
\end{tabular}

${ }^{a}$ Each of the four focal models (somatic symptoms, social impact, perceived causes, self-treatment at home) were adjusted for socio-demographic characteristics.

${ }^{\mathrm{b}}$ Logistic regression coefficient with $95 \%$ confidence interval.

c Bold values indicate $P \leqslant 0 \cdot 05$.

${ }^{d}$ Interaction with site: refers to rural compared to urban site, with urban site as the baseline. Site-specific effects on variables considered only if $P<0 \cdot 1$ for site-interaction term. $* P \leqslant 0 \cdot 05, * * P \leqslant 0 \cdot 01$.

${ }^{\mathrm{e}}$ Difference in corrected Akaike's Information Criterion [ $\left.\triangle(\mathrm{AICc})\right]$ between each model and the model with the lowest AICc. Comprehensive model (Table 4) had the lowest AICc and was assigned a value of zero. Models with lower $\triangle$ (AICc) values are considered better fitted than those with higher values. Bold values indicate models that are better than the model containing only socio-demographic characteristics.

f 'Other causes' refers to responses that could not be coded within designated categories of the interview. The variety of responses coded under 'other causes' included contact with infected persons, unprotected sexual intercourse, cold weather, mosquitoes, breathing in contaminated air and eating cold food.

$\mathrm{g}$ Variables with which each focal model was adjusted.

${ }^{\mathrm{h}}$ Compared with the occupation of agriculture. 
Table 3. Multivariate analysis (focal models) of socio-cultural determinants of anticipated oral cholera vaccine acceptance at the high price (US\$ 8.4) and assessment of models

\begin{tabular}{|c|c|c|c|c|}
\hline Focal models ${ }^{\mathrm{a}}$ & Coefficient $(95 \% \mathrm{CI})^{\mathrm{b}}$ & $P$ value $^{\mathrm{c}}$ & $\operatorname{Int}^{\mathrm{d}}$ & $\Delta(\mathrm{AICc})^{\mathrm{C}}$ \\
\hline Patterns of distress: somatic symptoms & & & & $11 \cdot 9$ \\
\hline Abdominal pain/discomfort & $0 \cdot 30(-0.01$ to 0.60$)$ & $0 \cdot 055$ & & \\
\hline Loss of appetite & $-0.63(-1.03$ to -0.23$)$ & $0 \cdot 002$ & & \\
\hline Weakness & $-0 \cdot 12(-0 \cdot 35$ to $0 \cdot 12)$ & $0 \cdot 319$ & & \\
\hline Palpitations & $0.07(-0.25$ to 0.39$)$ & $0 \cdot 669$ & & \\
\hline Confusion (urban site) & $0 \cdot 21(-0.41$ to 0.83$)$ & $0 \cdot 502$ & & \\
\hline Confusion (rural site) & $-1.02(-1.66$ to -0.39$)$ & $0 \cdot 002$ & $* *$ & \\
\hline Perceived causes & & & & $32 \cdot 22$ \\
\hline Other causes (urban site) ${ }^{\mathrm{f}}$ & $0 \cdot 08(-0 \cdot 30$ to $0 \cdot 46)$ & $0 \cdot 691$ & & \\
\hline Other causes (rural site) ${ }^{\mathrm{f}}$ & $-0.35(-0.63$ to -0.08$)$ & $0 \cdot 013$ & $\dagger$ & \\
\hline Self-treatment at home & & & & $24 \cdot 08$ \\
\hline Drinking more water or liquids & $0 \cdot 07(-0 \cdot 10$ to $0 \cdot 23)$ & $0 \cdot 421$ & & \\
\hline Herbal treatment & $-0.27(-0.48$ to -0.06$)$ & $0 \cdot 010$ & & \\
\hline Oral rehydration solution & $-0 \cdot 12(-0.26$ to 0.02$)$ & $0 \cdot 094$ & & \\
\hline Prayers & $0.43(0.11$ to $0 \cdot 76)$ & $0 \cdot 009$ & & \\
\hline Socio-demographics ${ }^{\mathrm{g}}$ & & & & $33 \cdot 54$ \\
\hline Primary school vs. no education & $0.58(-0.61$ to $1 \cdot 77)$ & $0 \cdot 339$ & & \\
\hline Secondary school $v s$. no education & $0.78(-0.43$ to 1.99$)$ & $0 \cdot 209$ & & \\
\hline Regular and dependable household income & $0 \cdot 72(0 \cdot 25$ to $1 \cdot 18)$ & $0 \cdot 002$ & & \\
\hline Household size & $-0.07(-0.17$ to 0.02$)$ & $0 \cdot 122$ & & \\
\hline Occupation: housewife, student, retired ${ }^{\mathrm{h}}$ & $-0 \cdot 12(-0 \cdot 80$ to $0 \cdot 55)$ & $0 \cdot 716$ & & \\
\hline $\begin{array}{l}\text { Occupation: self-employed, formally employed, } \\
\text { casual labour }^{\mathrm{h}}\end{array}$ & $0.02(-0.58$ to 0.62$)$ & $0 \cdot 950$ & & \\
\hline Gender (male vs. female) & $0.33(-0.12$ to 0.78$)$ & $0 \cdot 153$ & & \\
\hline Site (rural vs. urban) & $-0.34(-0 \cdot 87$ to $0 \cdot 20)$ & $0 \cdot 216$ & & \\
\hline
\end{tabular}

a Each of the three focal models (somatic symptoms, perceived causes, self-treatment at home) were adjusted for sociodemographic characteristics.

${ }^{\mathrm{b}}$ Logistic regression coefficient with $95 \%$ confidence interval.

c Bold values indicate $P \leqslant 0 \cdot 05$.

d Interaction with site: refers to rural compared to urban site, with urban site as the baseline. Site-specific effects on variables considered only if $P<0 \cdot 1$ for site-interaction term. $\dagger P<0 \cdot 1, * * P \leqslant 0 \cdot 01$.

${ }^{\mathrm{e}}$ Difference in corrected Akaike's Information Criterion [ $\triangle$ (AICc)] between each model and the model with the lowest AICc. Comprehensive model (Table 5) had the lowest AICc and was assigned a value of zero. Models with lower $\Delta$ (AICc) values are considered better fitted than those with higher values. Bold values indicate models that are better than the model containing only socio-demographic characteristics.

f 'Other causes' refers to responses that could not be coded within designated categories of the interview. The variety of responses coded under 'other causes' included contact with infected persons, unprotected sexual intercourse, cold weather, mosquitoes, breathing in contaminated air and eating cold food.

$\mathrm{g}$ Variables with which each focal model was adjusted.

h Compared with the occupation of agriculture.

positively associated while an increasing household size was negatively associated.

Site-specific interactions were not observed for any variables at the high price and were present for just one variable at the medium price.

\section{DISCUSSION}

Findings from this study indicate high levels of acceptance for an OCV among urban and rural residents in Western Kenya. Quantitative and narrative analysis showed that respondents perceive a general benefit from immunization. The extensive interest and demand for OCVs indicates a likelihood of good coverage during mass vaccination initiatives. The study also showed that $91.3 \%$ of the respondents considered the illness as very serious and $96 \cdot 3 \%$ believed that it had life-threatening consequences. The prospect of an effective vaccine campaign is further supported by this data given the widely acknowledged 
Table 4. Multivariate analysis (comprehensive model) of socio-cultural determinants of anticipated oral cholera vaccine acceptance at the medium price (US\$4.2)

\begin{tabular}{|c|c|c|}
\hline Explanatory variables & Coefficient $(95 \% \mathrm{CI})^{\mathrm{a}}$ & $P$ value ${ }^{\mathrm{b}}$ \\
\hline \multicolumn{3}{|l|}{ Patterns of distress: somatic symptoms } \\
\hline Bloody stool & $-0.29(-0.56$ to $-0 \cdot 01)$ & $0 \cdot 042$ \\
\hline Very thirsty & $0 \cdot 57(-0.01$ to $1 \cdot 15)$ & $0 \cdot 054$ \\
\hline Loss of appetite & $-0 \cdot 77(-1 \cdot 19$ to $-0 \cdot 34)$ & $<0 \cdot 001$ \\
\hline Confusion & $-0.54(-0.94$ to -0.13$)$ & 0.009 \\
\hline \multicolumn{3}{|l|}{ Perceived causes } \\
\hline Other causes ${ }^{\mathrm{c}}$ & $-0 \cdot 16(-0 \cdot 38$ to $0 \cdot 07)$ & $0 \cdot 166$ \\
\hline \multicolumn{3}{|l|}{ Self-treatment at home } \\
\hline Herbal treatment & $-0 \cdot 16(-0 \cdot 37$ to $0 \cdot 06)$ & $0 \cdot 153$ \\
\hline Prayers & $0.46(0.09$ to 0.82$)$ & $0 \cdot 015$ \\
\hline \multicolumn{3}{|l|}{ Socio-demographics } \\
\hline Primary school $v s$. no education & $0 \cdot 70(-0 \cdot 60$ to $2 \cdot 00)$ & $0 \cdot 291$ \\
\hline Secondary school vs. no education & $1 \cdot 37(0 \cdot 03$ to $2 \cdot 71)$ & $0 \cdot 045$ \\
\hline $\begin{array}{l}\text { Regular and dependable } \\
\text { household income }\end{array}$ & $0.93(0 \cdot 40$ to $1 \cdot 46)$ & $0 \cdot 001$ \\
\hline Household size & $-0 \cdot 10(-0 \cdot 21$ to $0 \cdot 01)$ & $0 \cdot 063$ \\
\hline
\end{tabular}

a Logistic regression coefficient with $95 \%$ confidence interval.

b Bold values indicate $P \leqslant 0 \cdot 05$.

c 'Other causes' refers to responses that could not be coded within designated categories of the interview. The variety of responses coded under 'other causes' were contact with infected persons, unprotected sexual intercourse, cold weather, mosquitoes, breathing in contaminated air and eating cold food.

Table 5. Multivariate analysis (comprehensive model) of socio-cultural determinants of anticipated oral cholera vaccine acceptance at the high price (US\$ 8.4)

\begin{tabular}{|c|c|c|c|}
\hline Explanatory variables & Coefficient $(95 \% \mathrm{CI})^{\mathrm{a}}$ & $P$ value ${ }^{\mathrm{b}}$ & $\operatorname{Int}^{\mathrm{c}}$ \\
\hline \multicolumn{4}{|l|}{ Patterns of distress: somatic symptoms } \\
\hline Abdominal pain/discomfort & $0 \cdot 27(-0 \cdot 04$ to $0 \cdot 58)$ & $0 \cdot 085$ & \\
\hline Loss of appetite & $-0 \cdot 64(-1 \cdot 04$ to $-0 \cdot 23)$ & $0 \cdot 002$ & \\
\hline Confusion (urban site) & $0 \cdot 19(-0.46$ to 0.83$)$ & $0 \cdot 568$ & \\
\hline Confusion (rural site) & $-1.08(-1.73$ to -0.42$)$ & $0 \cdot 001$ & $* *$ \\
\hline \multicolumn{4}{|l|}{ Self-treatment at home } \\
\hline Herbal treatment & $-0 \cdot 27(-0.48$ to -0.06$)$ & $0 \cdot 012$ & \\
\hline Oral rehydration solution & $-0 \cdot 11(-0 \cdot 25$ to $0 \cdot 03)$ & $0 \cdot 113$ & \\
\hline Prayers & $0.42(0.09$ to 0.74$)$ & $0 \cdot 013$ & \\
\hline \multicolumn{4}{|l|}{ Socio-demographics } \\
\hline Gender (male $v s$. female) & $0.39(-0.09$ to 0.87$)$ & $0 \cdot 107$ & \\
\hline Site (rural vs. urban) & $0 \cdot 73(-0.17$ to $1 \cdot 62)$ & $0 \cdot 111$ & \\
\hline Primary school vs. no education & $0 \cdot 95(-0 \cdot 41$ to $2 \cdot 31)$ & $0 \cdot 172$ & \\
\hline Secondary school vs. no education & $1 \cdot 28(-0 \cdot 12$ to $2 \cdot 67)$ & $0 \cdot 074$ & \\
\hline $\begin{array}{l}\text { Regular and dependable } \\
\text { household income }\end{array}$ & $0 \cdot 81(0 \cdot 31$ to $1 \cdot 30)$ & $0 \cdot 001$ & \\
\hline Household size & $-0 \cdot 11(-0 \cdot 21$ to $-0 \cdot 01)$ & $0 \cdot 031$ & \\
\hline
\end{tabular}

a Logistic regression coefficient with $95 \%$ confidence interval.

b Bold values indicate $P \leqslant 0 \cdot 05$.

c Interaction with site: refers to rural compared to urban site, with urban site as the baseline. Site-specific effects on variables considered only if $P<0 \cdot 1$ for siteinteraction term. $* * P \leqslant 0 \cdot 01$. 
observation that perceived severity of a disease is closely associated with likelihood of vaccine uptake $[10,28]$.

This study demonstrated that socio-cultural determinants explained anticipated vaccine acceptance better than socio-demographic factors alone. Identification of such socio-cultural determinants of OCV acceptance provides data relevant to ensure better coverage in an actual campaign. Past cholera control campaigns that have faced severe community resistance [29], and free treatment initiatives for other diseases that were rejected [30], further underscores the importance of paying attention to local sociocultural environments prior to interventions. Community studies are necessary to plan and prepare for vaccine campaigns [31]. This study provides an approach to integrate qualitative and quantitative empirical data, explain local cultural concepts of illness and guide disease control.

Several determinants of anticipated OCV acceptance were notable. Thirst, a cholera-specific symptom, was associated positively with acceptance. Non-specific physical symptoms for cholera were associated with a lower priority for the vaccine. Bloody stool, a characteristic symptom of other diarrhoeal illnesses, such as shigellosis, amoebic dysentery, campylobacteriosis, etc., was also negatively associated with OCV acceptance. The ability to discern cholera symptoms from symptoms of other diarrhoeal illnesses argues for a high level of awareness in the community. These findings also indicate that efforts to promote community awareness during control interventions need to highlight cholera-specific symptoms. Furthermore, given the definitive ideas of cholera possessed by the community, non-specific reference to diarrhoeal illness may lead to unreasonable expectations that the OCV will prevent all diarrhoea, leading to disappointment and possible discrediting of a useful vaccine.

Education was a predictor of vaccine acceptance. Interestingly, health education was reported as the most useful method of preventing cholera by the majority of respondents, and was frequently requested. A similar finding was reported from a study in Pakistan where education and knowledge about vaccines were associated with vaccine uptake [32]. These findings highlight the value of education and promoting health awareness in cholera control. However, at the high price, secondary school education was no longer significantly associated with OCV acceptance, indicating that education too has the ability to influence acceptance only to a certain extent. Above a certain price, economic factors may play a more prominent role in influencing OCV acceptance.

At the high price, self-help with herbal treatment was a significant negative determinant of acceptance. It appears that higher cost of the vaccine makes alternative, less expensive forms of treatment preferable. This finding is consistent with other literature in Kenya noting that the high cost of conventional Western drugs often makes them inaccessible, thereby promoting reliance on traditional remedies [33]. Pluralistic health-seeking practices, including traditional remedies, are widely used in other African countries [34]; however, in our study they appear to compete with biomedical interventions, especially when the higher cost of vaccine becomes a barrier.

The priority of prayer, on the other hand, was complementary to vaccine interventions - an additional, rather than alternative source of help. Other studies suggest religious beliefs may be antagonistic to vaccine intervention $[35,36]$. A study in Benin found that vaccination was rejected in some religious communities because they believed that they 'require only prayer to protect and heal them in times of illness' [37]. The finding in Kenya, that reporting self-help with prayer was significantly associated with OCV acceptance, suggests a possible role played by religious institutions in encouraging the use of biomedicine. Although religious sectarian differences may influence the perceived benefits of medical interventions, we found no differences in anticipated OCV acceptance in members of the Legio Maria church, which some studies suggest may promote faith healing and reject biomedicine [38], and members of other church groups.

Financial viability, based on reporting a regular household income and a smaller household size, influenced OCV acceptance. While this finding is what would be expected for relatively high-priced vaccines, it underscores the importance of keeping costs reasonable. Furthermore, as the price of OCV was increased, determinants that had influenced vaccine acceptance at a lower price, such as education, were no longer relevant. The increasing price levels of OCV were introduced to provide an indication of priority and demand for the vaccine. Findings suggest that regardless of priorities and commitment to obtain an OCV, above a certain price it was simply beyond the means of many. In this study, the high price was the threshold. Hence, a full-cost recovery model with Dukoral may not be considered in this 
setting. However, it may be considered for OCVs that can be produced at a far lower cost, such as Shanchol [39].

Analysis of OCV acceptance did not reveal significant site-specific determinants; socio-cultural factors influencing OCV acceptance for urban and rural residents were similar. However, enthusiasm for an OCV was significantly higher in urban than rural respondents at the medium and high prices. This may be explained by the presence of greater disposable income and better education as has been observed in another vaccine study [32] or by higher perceived risk and vulnerability to the disease. In this study, both hypotheses remain plausible, as the urban respondents have better incomes and education than their rural counterparts. They also may attach a greater priority to receiving an OCV given the more crowded and unsanitary conditions that they have to contend with.

The main limitation of this study is the ability to relate anticipated acceptance with actual acceptance in the context of a vaccine campaign. Recognizing that there is a difference between what people say and what they actually do [40] anticipated acceptance may not perfectly guide actual acceptance. Inasmuch as this study provided a community assessment of vaccine demand and findings on predictors of $\mathrm{OCV}$ acceptance which support reasonable expectations (e.g. secondary school education was a predictor for OCV acceptance), further research addressing the nature of the relationship between anticipated and actual acceptance is needed. It also remains to be seen whether the predictors of anticipated OCV acceptance would remain significant in the context of an actual mass vaccination campaign.

Further research could include an assessment of whether findings from this study may be generalized across other settings. At some level we expect broad similarities in factors influencing OCV acceptance; however, particular priorities may be culture-specific. It would be fruitful to develop a framework for vaccine acceptance by conducting more such studies in different settings to explain common features and context-specific differences.

In conclusion, this study found high levels of interest for an OCV in community residents in Western Kenya, although vaccine cost was revealed as a critical consideration. Socio-cultural factors played an important role in anticipated OCV acceptance and specific determinants were identified. This research also provides an approach for the study of socio-cultural determinants and barriers to vaccine acceptance in other settings.

\section{ACKNOWLEDGEMENTS}

We gratefully acknowledge the participation of the study population and the commitment of research assistants who conducted interviews. We thank public health officials from Siaya and Kisumu districts for providing cholera incidence data and other essential information. We thank Leticia Grize and Christian Schindler for valuable statistical support. This study was supported by funds from the Bill and Melinda Gates Foundation that is also gratefully acknowledged.

C.-L. Chaignat and R. Hutubessy are staff members of the World Health Organization. The authors alone are responsible for the views expressed in this publication and they do not necessarily represent the decisions, policy or views of the World Health Organization.

\section{DECLARATION OF INTEREST}

None.

\section{REFERENCES}

1. Lopez AD, et al. Global and regional burden of disease and risk factors, 2001: systematic analysis of population health data. Lancet 2006; 367: 1747-1757.

2. Zuckerman JN, Rombo L, Fisch A. The true burden and risk of cholera: implications for prevention and control. Lancet Infectious Diseases 2007; 7 : 521-530.

3. WHO. Cholera, 2009. Weekly Epidemiological Record $2010 ; 85: 293-308$.

4. WHO. Cholera vaccines: WHO position paper. Weekly Epidemiological Record 2010; 85: 117-128.

5. WHO, UNICEF. Meeting the MDG water and sanitation target: the urban and rural challenge of the decade. Geneva: WHO, 2006.

6. Global Task Force on Cholera Control, WHO. Oral cholera vaccine use in complex emergencies: what next? Report of a WHO meeting, 14-16 December 2005, Cairo, Egypt. Geneva: World Health Organization, 2006; Report no. WHO/CDS/NTD/IDM/2006.2.

7. Lucas ME, et al. Effectiveness of mass oral cholera vaccination in Beira, Mozambique. New England Journal of Medicine 2005; 352: 757-767.

8. Sur D, et al. Efficacy of a low-cost, inactivated wholecell oral cholera vaccine: results from 3 years of followup of a randomized, controlled trial. PLoS Neglected Tropical Diseases 2011; 5: e1289.

9. Larson HJ, et al. Addressing the vaccine confidence gap. Lancet 2011 ; 378: 526-535. 
10. Stanton BF. Assessment of relevant cultural considerations is essential for the success of a vaccine. Journal of Health, Population and Nutrition 2004; 22: 286-292.

11. Raude J, Caille-Brillet AL, Setbon M. The 2009 pandemic H1N1 influenza vaccination in France: who accepted to receive the vaccine and why? PLoS Currents 2010; 2: RRN1188.

12. Mortensen GL. Drivers and barriers to acceptance of human-papillomavirus vaccination among young women: a qualitative and quantitative study. $B M C$ Public Health 2010; 10: 68.

13. Islam $\mathbf{Z}$, et al. Private demand for cholera vaccines in rural Matlab, Bangladesh. Health Policy 2008; 85: 184-195.

14. Kim D, et al. Private demand for cholera vaccines in Hue, Vietnam. Value in Health 2008; 11: 119-128.

15. Lucas ME, et al. Private demand for cholera vaccines in Beira, Mozambique. Vaccine 2007; 25: 2599-2609.

16. DeRoeck D, et al. Policymakers' views regarding the introduction of new-generation vaccines against typhoid fever, shigellosis and cholera in Asia. Vaccine 2005; 23 : 2762-2774.

17. Ali M, et al. Community participation in two vaccination trials in slums of Kolkata, India: a multi-level analysis. Journal of Health, Population and Nutrition 2010; 28: 450-457.

18. Feikin DR, Tabu CW, Gichuki J. Does water hyacinth on East African lakes promote cholera outbreaks? American Journal of Tropical Medicine and Hygiene $2010 ; 83$ : 370-373.

19. Shapiro RL, et al. Transmission of epidemic Vibrio cholerae $\mathrm{O} 1$ in rural western Kenya associated with drinking water from Lake Victoria: an environmental reservoir for cholera? American Journal of Tropical Medicine and Hygiene 1999; 60: 271-276.

20. Shikanga OT, et al. High mortality in a cholera outbreak in western Kenya after post-election violence in 2008. American Journal of Tropical Medicine and Hygiene 2009; 81: 1085-1090.

21. Weiss MG. Cultural epidemiology: an introduction and overview. Anthropology and Medicine 2001; 8: 5-29.

22. UN-HABITAT. Cities without slums: situational analysis of informal settlements in Kisumu. Nairobi: United Nations Human Settlements Programme (UNHABITAT), 2005; Report no. HS/783/05E.

23. Olago D, et al. Climatic, socio-economic, and health factors affecting human vulnerability to cholera in the Lake Victoria basin, East Africa. Ambio 2007; 36: 350-358.

24. Ministry of public health and sanitation. Siaya district health profile. Siaya, Kenya: Health Records Office, 2009.

25. Schaetti C, et al. Oral cholera vaccine use in Zanzibar: socioeconomic and behavioural features affecting demand and acceptance. BMC Public Health 2009; 9: 99 .
26. Weiss MG. Explanatory Model Interview Catalogue (EMIC): framework for comparative study of illness. Transcultural Psychiatry 1997; 34: 235-263.

27. Schaetti C, et al. Social and cultural features of cholera and shigellosis in peri-urban and rural communities of Zanzibar. BMC Infectious Diseases 2010; 10: 339.

28. Teitler-Regev S, Shahrabani S, Benzion U. Factors affecting intention among students to be vaccinated against A/H1N1 influenza: a health belief model approach. Advances in Preventive Medicine 2011; 2011: 353207.

29. Nations MK, Monte CM. 'I'm not dog, no!' : cries of resistance against cholera control campaigns. Social Science \& Medicine 1996; 43: 1007-1024.

30. Parker M, Allen T, Hastings J. Resisting control of neglected tropical diseases: dilemmas in the mass treatment of schistosomiasis and soil-transmitted helminths in north-west Uganda. Journal of Biosocial Science 2008; 40: 161-181.

31. Kaljee LM, et al. Sociobehavioural research methods for the introduction of vaccines in the Diseases of the Most Impoverished Programme. Journal of Health, Population and Nutrition 2004; 22: 293-303.

32. Mitchell S, et al. Equity and vaccine uptake: a crosssectional study of measles vaccination in Lasbela District, Pakistan. BMC International Health and Human Rights 2009; 9 (Suppl.1): S7.

33. Matu EN, vanStaden J. Antibacterial and anti-inflammatory activities of some plants used for medicinal purposes in Kenya. Journal of Ethnopharmacology 2003; 87: 35-41.

34. Cassell JA, et al. The social shaping of childhood vaccination practice in rural and urban Gambia. Health Policy and Planning 2006; 21 : 373-391.

35. Ruijs WL, et al. Religious subgroups influencing vaccination coverage in the Dutch Bible belt: an ecological study. BMC Public Health 2011; 11: 102.

36. Jegede AS. What led to the Nigerian boycott of the polio vaccination campaign? PLoS Medicine 2007; 4: e73.

37. Fourn L, et al. Determinants of parents' reticence toward vaccination in urban areas in Benin (West Africa). BMC International Health and Human Rights 2009; 9 (Suppl.1): S14.

38. Prince R. The Legio Maria church in Western Kenya: healing with the Holy Spirit and the rejection of medicines (dissertation). London, UK: University College London, 1999, 72 pp.

39. Ad-hoc Cholera Vaccine Working Group, WHO. Background paper on the integration of oral cholera vaccines into global cholera control programmes presented to the WHO SAGE. (http://www.who.int/ immunization/sage/1_Background_Paper_Cholera_ Vaccines_FINALdraft_13_oct_v2.pdf), 2009. Accessed 1 February 2012.

40. Mpazi VM, Mnyika KS. Knowledge, attitudes and practices regarding cholera outbreaks in Ilala municipality of Dar es Salaam region, Tanzania. East African Journal of Public Health 2005; 2: 6-11. 